\title{
Justicia, capacidades diversas y acceso igualitario a las tecnologías de la información y comunicación: el impacto de la desigualdad relativa en el cuidado de la salud
}

\author{
Justice, diverse capabilities and equal access to information and \\ communication technologies: the impact of relavite inequality \\ on health care
}

\begin{abstract}
Resumen
El objetivo del presente trabajo es analizar el marco filosófico de justicia social (SenNussbaum) que se aplica en derechos humanos para fundamentar el acceso igualitario a las tecnologías de la información y comunicación (TIC) y tecnologías asistivas (TA) de personas con capacidades diversas, considerado este acceso como uno de los determinantes sociales del derecho a la salud. La hipótesis que inspira esta investigación tiene como eje subrayar que el no acceso igualitario a TIC y a TA origina - en la mayoría de los casos - no sólo la misma situación de 'discapacidad' por entorno social desfavorable, sino que también incide en la salud psicosocial del citado grupo como consecuencia del estrés crónico generado por la desigualdad relativa. Este enfoque social integral, vincula las necesidades individuales, familiares y del medio ambiente con el fin de identificar mejor los problemas complejos de inclusión y acceso a la salud de las personas y/o grupos en sociedades no igualitarias, para no descuidar las enfermedades psicosociales producto de diferencias sociales y estigmatizaciones. Se trata de brindar un marco de justicia social en políticas públicas integrales e interseccionales, que tenga en cuenta las implicancias del acceso y uso social adaptado de TIC en personas con capacidades diversas en sociedades no igualitarias.
\end{abstract}

\section{Palabras clave}

Teorías de justicia, discapacidad, tecnologías de la información y comunicación (TIC), tecnologías asistivas (TA), determinantes psicosociales, desigualdad absoluta, desigualdad relativa, salud/enfermedad.

\footnotetext{
Abstract

The aim of the present paper is to analyze the philosophical framework of social justice (Sen-Nussbaum) applied in human rights to support the equal access to information and communication technologies (ICT) and assistive technologies (AT) of people with divers abilities, considering this access as one of the social determinants of the right to health. The hypothesis that inspires this research is to emphasize that non-equal access to ICT and AT causes - in most cases - not only the same situation of 'disability' due to unfavorable social environment, but also affects psychosocial health of the aforementioned group as a consequence of chronic stress generated by relative inequality. This integral social approach links the individual, family and environmental needs in order to better identify the complex problems of inclusion and access to health care of people and/or groups in unequal societies, so as not to neglect the psychosocial diseases product of social differences and stigmatization. The aim is to provide a framework of social justice in integral and intersectional public policies that take into account the implications of access and adapted social use of ICT in people with diverse abilities in non-equal societies.

\section{Keywords}

Justice theories, disability, information and communication technologies (ICT), assistive technologies (AT), psicosocial determinants, absolute inequality, relative inequality, health/disease.
}

\section{María Graciela de Ortúzar} $<$ mariagracieladeortuzar@gmail. com>

Universidad Nacional de La Plata. CONICET. Universidad Nacional de la Patagonia Austral. Argentina

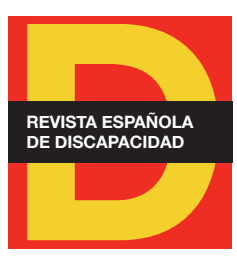

Para citar:

De Ortúzar, M. G. (2018): “Justicia, capacidades diversas y acceso igualitario a las tecnologías de la información y comunicación (TIC): el impacto de la desigualdad relativa en el cuidado de la salud". Revista Española de Discapacidad, 6 (I): 91 111.

Doi: <https://doi.org/10.5569/23405104.06.01.05>

Fecha de recepción: 17-06-2016 Fecha de aceptación: 10-03-2018

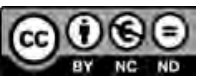




\section{Introducción ${ }^{1}$}

El objetivo del presente trabajo es analizar el marco filosófico de justicia social (Sen-Nussbaum) que se aplica a la defensa de dos derechos humanos básicos para las personas con capacidades diversas: el derecho al conocimiento/acceso igualitario a las tecnologías de la información y comunicación (TIC) y el derecho a la salud. La hipótesis que sostendré aquí es que la falta de acceso igualitario a las TIC conlleva no sólo la desigualdad absoluta en personas con capacidades diversas (discapacidad por entorno social o por falta de prevención oportuna). También genera un tipo especial de desigualdad relativa ${ }^{2}$, responsable del estrés crónico y factor clave de la salud psicosocial, la cual surge a partir de las diferencias sociales establecidas en el acceso a las TIC. Su evaluación es central para el desarrollo de un marco amplio, integral e interseccional de justicia social en políticas públicas de salud para personas con capacidades diversas en sociedades no igualitarias y/o regiones aisladas.

La atención de la salud como derecho humano exige el acceso a los avances en las ideas, técnicas o conocimientos (bienes públicos) que han sido históricamente desarrollados por la humanidad en diferentes sociedades, y que deberían ser aplicados de acuerdo a las necesidades y/o prioridades en cada región. A pesar del reconocimiento de este derecho, existe una importante brecha digital social entre países y entre grupos al interior de los mismos (De Ortúzar, 2014). El no acceso igualitario a TIC constituye, también, un determinante social de la salud (Filho, 2001). Este responde - a nivel global - a la imposición del Acuerdo sobre los aspectos del Derecho de Propiedad Intelectual relacionados con el comercio (en adelante ADPIC) desde la Organización Mundial de Comercio (en adelante OMC), el cual entra en conflicto con el derecho a la salud (De Ortúzar, 2003). De acuerdo a Filho (2001: 409), "las inequidades de información son un importante determinante de las inequidades en salud... La superación de dichas inequidades de acceso a la información, particularmente a través del uso de las nuevas TIC, puede significar un importante avance en el sentido de garantizar a todos el derecho a la salud".

Ante esta situación, las desigualdades en el acceso a TIC pueden repercutir de diversas maneras en el bienestar general de las poblaciones, y muy particularmente en el bienestar y/o salud de grupos con capacidades diversas. Por una parte, el acceso igualitario para aquellos que más se beneficiarían en el uso social de TIC es una necesidad básica. Un caso particularmente relevante es el acceso a TIC de personas con capacidades diversas en poblaciones aisladas - acceso a telemedicina, adaptación del entorno educativo y social, movilidad adaptada, entre otros - (De Ortúzar, 2012). En segundo lugar, esta desigualdad absoluta puede generar desigualdades relativas en salud para el grupo por diferenciación en el acceso (Wilkinson y Picket, 2009; Sen, 2011), provocando estrés crónico y nuevas enfermedades psicosociales. Las diferencias

1. Una versión preliminar y parcial de este trabajo ha sido expuesta en el 'Simposio Argentino de Informática y Derecho (SID)' en agosto de 2016 y publicada en Actas. El presente artículo ha sido realizado en el marco la Beca de Investigación en la Universidad Complutense de Madrid (FC), en representación de la Universidad Nacional de la Patagonia Austral (UNPA) y de los respectivos proyectos de investigación del Instituto de Investigación de Humanidades y Ciencias Sociales (IdlHCS), del Consejo Nacional de Investigaciones Científicas y Tecnológicas (CONICET) y de la Secretaría de Ciencia y Técnica (SECYT) de la Universidad Nacional de La Plata (UNLP) en Argentina. Agradezco a las instituciones citadas por el apoyo recibido. En otro orden, quiero agradecer el aporte enriquecedor de los evaluadores anónimos de la revista a la temática planteada y las oportunas sugerencias, especialmente sobre el marco filosófico.

2. La desigualdad relativa se define a partir de las diferencias de estatus percibidas socialmente, que pueden ser consecuencia indirecta de la misma desigualdad absoluta (falta de satisfacción de necesidades básicas). También puede originarse por las diferencias de ingresos relativos que generan las 'diferencias sociales' (esto es, existe satisfacción de necesidades básicas pero persiste la desigualdad relativa que origina nuevas enfermedades psicosociales). 
sociales generan no sólo diferencias de acceso a TIC sino también diferencias de estatus social vinculadas al acceso a TIC, constituyendo éstas uno de los determinantes principales de la salud (desigualdad relativa social) ${ }^{3}$.

La salud no puede comprenderse si la miramos sólo como un fenómeno biológico bajo el microscopio. La salud de las poblaciones depende, en gran parte, de la interacción entre la herencia biológica y su contexto social o formas de vida (epigenética). Al respecto, Wilkinson y Pickett (2009) resaltan cómo las desigualdades sociales constituyen los determinantes psicosociales principales que afectan nuestra salud en la sociedad actual. En la misma línea, Breihl (2013: 16) considera que la salud se relaciona con la reproducción social y los modos de vivir, de enfermar y de morir de cada sociedad. En consecuencia, un primer punto a resaltar es la necesidad de evaluar el acceso igualitario a la salud de personas con capacidades diversas, considerando el impacto del acceso a TIC para este grupo, pero evaluando a la salud no exclusivamente desde el punto de vista biológico o tecnológico, sino teniendo en cuenta los diversos determinantes sociales que inciden en la salud y considerando a la misma como un proceso dinámico, relacional y diverso.

Para la fundamentación de la importancia conjunta de la evaluación de la desigualdad relativa en acceso a TIC de personas con capacidades diversas, comenzaré analizando la definición de 'capacidades diversas/ discapacidad' y sus implicancias en los criterios de justicia en salud. En segundo lugar, plantearé las ventajas y desventajas del marco legal vigente y sus problemas de aplicación en nuestra región. En tercer lugar, presentaré la fundamentación filosófica desde teorías de justicia, mostrando la deuda social existente hacia las personas con capacidades diversas en 'sociedades no igualitarias'4, y, especialmente en zonas aisladas. En último lugar, mostraré por qué es importante no desatender en políticas públicas el problema de acceso a TIC y las desigualdades relativas que este genera, con claras consecuencias en la salud psicosocial de las personas con capacidades diversas. Como propuesta final se sugiere la incorporación de una evaluación integral e interseccional de determinantes sociales en salud, la cual contrasta con la actual evaluación aislada de tecnologías sanitarias. Se promueve así una mejor identificación de los problemas complejos de salud de personas con capacidades diversas en países y regiones no igualitarias, con el fin de contribuir a una fundamentación armónica, coherente y operativa en los marcos legales para la regulación del uso social de TIC en grupos vulnerables.

\footnotetext{
3. En relación al uso de TIC, en este punto es importante distinguir: a) el uso social citado de esta tecnología, y b) el uso 'light' de TIC, el cual responde exclusivamente a los intereses de mercado, existiendo un abanico de ofertas comerciales de tecnologías de punta para personas con capacidades diversas. Esto último, muchas veces, sólo lleva a complejizar sus vidas y no responde a sus necesidades. Por ejemplo, por una parte uno podría argumentar que no puede imponerse, como una obligación social, el uso de miembros ortopédicos electrónicos. El individuo es libre de desarrollar otras capacidades sin los mismos y, en este sentido, puede optar por no utilizarlos. No obstante, es importante diferenciar entre: 1- la necesidad de no imponer el uso de la última tecnología de punta, bajo presión social y de mercado, 2- el hecho de no poder acceder a piernas ortopédicas que permitan a la persona integrarse socialmente. El no poder elegir constituye una gran diferencia social para dicha persona (véase al respecto De Ortúzar, 2012).

4. Si bien las sociedades igualitarias y no igualitarias constituyen modelos puros en el marco de los debates sobre justicia social, la distinción entre las mismas es relevante para la evaluación de la desigualdad relativa. A grandes rasgos, entendemos aquí por sociedades igualitarias aquellas en las que los ciudadanos acceden a la satisfacción de las necesidades básicas, siendo justa la distribución de las cargas y beneficios de acuerdo a las mismas, lo cual produce una tendencia a la igualdad y una disminución de las diferencias sociales. Por otra parte, las sociedades no igualitarias se caracterizan por una gran brecha entre los ciudadanos (ricos vs. pobres), la cual profundiza las diferencias sociales, no pudiendo satisfacer igualitariamente los ciudadanos las necesidades básicas.
} 


\section{Marco conceptual}

La definición de discapacidad ${ }^{5}$ posee importantes consecuencias en justicia distributiva social y sanitaria. Asimismo, de cómo definamos la salud/enfermedad depende también la especificación de los criterios de acceso a la atención de la salud. Por ello, en esta sección analizaremos los modelos históricos-conceptuales sobre el tema y estudiaremos sus implicancias en justicia social, políticas públicas en salud y acceso a $\mathrm{TIC}$ de personas con capacidades diversas.

\subsection{Modelo médico rehabilitador}

En primer modelo, el modelo médico rehabilitador, establece como criterio de salud/enfermedad la funcionalidad y la normalidad natural. Para este modelo los 'anormales funcionales' son invisibles por reclusión o confinamiento médico, generando importantes barreras culturales y de integración social. De acuerdo a la Clasificación Internacional del Funcionamiento (en adelante CIF), la discapacidad se reduce a un problema de la persona causado por enfermedad, trauma o condición, y requiere cuidados individuales ${ }^{6}$ para adaptar la persona a la situación (CIF, 2001). Según Palacios, el tratamiento que reciben las personas con discapacidad - desde este modelo- genera subestimación y discriminación, como así también dependencia (Palacios, 2008). En lo que hace a la igualdad y políticas públicas, el acceso a la salud tiene por finalidad compensar la anormalidad biológica individual, pero no implica contribuir a su integración social como ciudadano. Por el contrario, el perfil individual es utilizado para justificar el montaje de una abundante oferta comercial de productos tecnológicos.

\subsection{Modelo social de discapacidad}

El enfoque social, vigente a nivel formal en Argentina mediante la Convención sobre los Derechos de las Personas con Discapacidad ratificada por la Ley 26378 con jerarquía constitucional y por la Ley 27044 , hace hincapié en que la noción de discapacidad se centra en las limitaciones de la propia sociedad, no pudiendo escindirse del entramado social y familiar. Se sostiene que el concepto de discapacidad es variable de acuerdo a épocas y culturas, pero el tratamiento del problema siempre requiere modificaciones en el contexto ambiental, político y cultural para superar las barreras en la integración y lograr la participación en la vida política y social de todo ciudadano.

Propone, a nivel de políticas, el diseño universal y la accesibilidad. Eso es, requiere que todos los productos, entornos, procesos, bienes y servicios cumplan con los requisitos necesarios para poder ser utilizados por todas las personas de la forma más autónoma posible. Promueve la educación inclusiva, desafiando la normalidad impuesta sobre una realidad donde sólo existe diferencia (Palacios, 2008), y denunciando el aislamiento relacional. Así, el paradigma de protección integral enfatiza la interdependencia de derechos y

5. Para la profundización de las discusiones conceptuales sobre el término discapacidad véase Sherlaw, W. et al. (2013). Para la vinculación entre ciudadanía y discapacidad véase Díaz Velázquez (2010). Y para la profundización de los problemas éticos que pueden surgir en los diferentes modelos de justicia y discapacidad véase Canimas (2015).

6. Cursiva de la autora. 
la participación de la comunidad. En este camino, no podemos dejar de mencionar el rol central de la universidad, donde se trabaja para la integración social a través de la creación de software especial e inclusión de grupos con capacidades diversas en los ciber-centros educativos ${ }^{7}$, adaptación de juguetes para niños con capacidades diversas o adaptación de infraestructura, entre otros.

No obstante, en Argentina, como en muchos lugares del mundo, coexisten en la práctica ambos modelos, el modelo rehabilitador y el modelo social, existiendo conflictos de derechos en el momento de su aplicación.

\subsection{Modelo de la diversidad funcional}

Hace unas décadas atrás ha surgido un nuevo modelo, denominado modelo de la diversidad funcional, el cual propone sustituir el paradigma médico rehabilitador y superar también el paradigma social. Este nuevo modelo subraya que el concepto mismo de discapacidad es estigmatizador ${ }^{8}$. Pero en el momento de determinar cuáles sería los criterios de igualdad de acceso y distribución de la tecnología, el citado modelo no brinda criterios claros.

Uno podría preguntarse si debemos intervenir en la corrección de deficiencias o sólo considerarlas un signo de diversidad. Lo paradójico de este paradigma es sostener que la lucha debe darse en el nivel de interpretación de lo que consideramos deficiencia. Aquí creo que se confunden dos conceptos diferentes. De acuerdo con Canimas, una cosa es distinguir entre discapacidad - como concepto discriminador - y otra es negar la deficiencia misma (Canimas, 2015). Si sostenemos que es solo una diversidad, entonces no haremos nada para evitarlas, tratarlas, prevenirlas, mejorarlas. Existiendo conocimientos y tecnología para ello (ejemplo: test prenatales igualitarios que permiten intervenir en el seno materno en cardiopatías o en espina bífida, entre otros), tenemos obligaciones morales de actuar y de que esta tecnología esté al acceso de todos, para evitar que las deficiencias y enfermedades las sufran sólo los más pobres. De acuerdo con Canimas (2015: 94),

"La ciencia y la tecnología constituyen instrumentos necesarios para combatir el déficit y las disfunciones (pero los seguidores del modelo de la diversidad niegan, en un sentido, la aplicación de la tecnología - test genéticos-, aun cuando lo exigen en otro - ajustes del entorno-)".

En otras palabras, ¿cómo justificar la prioridad de investigar en personas con diversidad funcional si ésta no es ni mejor ni peor que otras? El modelo de la diversidad, como bien señala el autor citado anteriormente, no puede responder ésta pregunta.

Siguiendo a Canimas, considero que las deficiencias - no poder escuchar, no poder caminar, no poder ver, entre otras - hay que corregirlas si disponemos de tecnología para ello. Poder escuchar Mozart, el canto de los pájaros al amanecer, el latido del corazón del bebé, es algo que desearíamos que nuestros hijos pudieran

\footnotetext{
7. De acuerdo con Alaniz et al. (2010: 214), "las TIC pueden mitigar los problemas de niños con discapacidades, al permitir compensar deficiencias serias de los órganos sensoriales y del aparato motor. En casos de niños con sordera o deficiencias auditivas, así como ceguera y deficiencias visuales, las TIC se usan para mejorar el funcionamiento del órgano debilitado o servir como sustituto de uno inoperante".

8. En el presente trabajo hemos elegido utilizar el concepto de capacidades diversas, exceptuando cuando la cita corresponda a otro autor o a un modelo específico que remita a discapacidad.
} 
hacer $^{9}$. Si el conocimiento y la tecnología permiten evitar, corregir o paliar la deficiencia, entonces debemos disponer universalmente de esa tecnología para prevenir el daño.

Por lo tanto, el concepto de capacidades diversas es políticamente correcto y no conlleva la carga peyorativa y estigmatizadora de la discapacidad. Pero la interpretación del modelo hermenéutico de que toda deficiencia es sólo diversidad no es posible aplicarla en justicia en salud porque no permite esclarecer un criterio de prioridades en investigación y políticas sanitarias, al no brindar criterios claros de acceso a la salud.

\subsection{Modelo biopsicosocial}

Existe una interesante variante al modelo social, el paradigma biopsicosocial, el cual distingue entre la deficiencia y la discapacidad ignorada anteriormente, al mismo tiempo que tiene en cuenta la interacción de las dos dimensiones. La deficiencia es considerada una anomalía en las funciones o estructuras corporales que dificulta o impide realizar acciones que se consideran valiosas. La discapacidad es la interacción de esa deficiencia con el mundo, puede o no ser una discapacidad según quién y dónde se dé. La igualdad en capacidades diversas dependerán tanto del grado de acceso a TIC como de su uso social y aplicación integral en un entorno que fomente la equidad ${ }^{10}$.

El aporte del enfoque biopsicosocial se centra en señalar la importancia de prever las deficiencias, además de actuar en el entorno social (entramado social y dimensión afectiva familiar), siendo ambos criterios relevantes para políticas de salud. Este modelo concuerda con el enfoque social, sin dejar de garantizar -a todos los que lo necesiten - el acceso a la tecnología de acuerdo a sus necesidades.

\subsection{Conclusión parcial del marco conceptual}

Más allá del análisis teórico de los modelos, el problema práctico de fondo es la convivencia paradójica entre el modelo médico rehabilitador y el modelo social, el cual parecería olvidado en sociedades no igualitarias, donde el Estado se encuentra cada vez más ausente. El modelo rehabilitador continúa reinando en la práctica médica por ser funcional, en su perfil individual, al mercado de salud. Las vidas de las personas con capacidades diversas transcurren, en su mayoría, encerradas en su propio hogar - por falta de acceso a TIC, inclusión social, accesibilidad, educación y/o trabajo-. En el mejor de los casos son recluidas en las instituciones especiales creadas para su cuidado.

Los obstáculos para su integración social son materiales y simbólicos, y pueden ser percibidos tempranamente, por ejemplo cuando se les dificulta el acceso a su educación inicial si los jardines maternales o escuelas no han adaptado la infraestructura para la accesibilidad de los niños, falta de apoyo, entre otros). Esto ocurre porque la educación especial no ha sido desterrada de nuestra región, siendo justificada por carencias sociales, de infraestructura, de formación interdisciplinaria, por número de alumnos o por cuestiones edilicias y tecnológicas $-\mathrm{TIC}-$. Se aduce que la integración no implica que no sea necesario separarlos

9. Existen debates variados sobre este tema, como es el caso de la cultura de la sordera y otras comunidades. Véase al respecto Green (1997). 10. Un ejemplo que se ha dado en los debates sobre enfermedad es el caso de la dislexia, que desde la sociedad agraria no sería considerada una enfermedad por no impedir el normal desarrollo de este individuo en su sociedad, pero esto puede ser cuestionado. El punto es cuál es el criterio de bienestar para el desarrollo de dichas capacidades. 
en escuelas especiales porque esto conllevaría el cierre de éstas escuelas, y son necesarias (Alaniz et al., 2010) ${ }^{11}$.

El enfoque médico, el cual condena al aislamiento individual, entra en conflicto con el enfoque social, no reconociendo la estigmatización que conlleva esta separación. Asimismo, la discriminación no es solo a nivel educativo. Es común también en el transporte público, en los lugares de turismo, en los medios de comunicación, en los relatos históricos, entre otros. De acuerdo a Vallejos (2012: 201), las políticas específicas de compensación individual (pases libre de transporte, pensiones asistenciales, exención impositiva, educación especial) sólo muestran la persistencia del modelo médico rehabilitador por sobre el modelo social integrador.

El modelo social es ignorado por el Estado y los organismos internacionales, desconociéndose la necesidad de traducir el derecho en oportunidades para todos. Como podemos observar ${ }^{12}$, la deuda social en el acceso a TIC de personas con capacidades diversas es tan grande que hace que esta diversidad sea visible en las "sociedades igualitarias" pero no en las "sociedades no igualitarias". En las primeras, el acceso a TIC y TA permite la adaptación del entorno social (infraestructura y movilidad, entre otros), factor material indispensable con el desarrollo simultaneo de una política cultural de inclusión e integración social (factor cultural y simbólico). En este accionar conjunto se fomenta la mayor autonomía de dichas personas como ciudadanos activos. Sin embargo, las personas con capacidades diversas de países pobres o sociedades no igualitarias continúan siendo 'invisibles' para su propia comunidad y para el mundo siendo recluidas, en muchos casos, en sus hogares o en instituciones especiales.

\section{Marco legal existente}

De acuerdo con la UNESCO, es imprescindible entender que la convivencia paradójica de diferentes paradigmas, conjuntamente con el desconocimiento sobre el tema por parte de las autoridades y de la sociedad general, constituyen obstáculos para la integración y la inclusión de las personas con capacidades diversas. La armonización de normas y políticas de acuerdo a los avances de la citada Convención sobre los Derechos de las Personas con Discapacidad, implica trabajar para la integración y la justicia social contrarrestando la invisibilidad de los que sufren por el desconocimiento de sus derechos y las restricciones de acceso a TIC (De Ortúzar, 2016) a partir de las diferencias de ingresos.

\footnotetext{
11. Según Alaniz et al. (2010: 214): “... en nuestro caso la integración de los alumnos en escuelas comunes se realiza con el acompañamiento y seguimiento permanente de la Escuela Especial a través de una docente integradora y reuniones periódicas de los docentes con el equipo psicopedagógico de la Escuela Especial. Por otro lado, los alumnos asisten a clases en la Escuela Especial a contra turno".

12. Al respecto, uno de las motivaciones principales para escribir sobre este tema ha sido la fuerte vivencia sobre el citado contraste en la visibilidad de las personas con capacidades diversas en espacios públicos percibido por la autora en la oportunidad de una instancia de investigación en Montreal (Canadá), la cual contrastaba con sus estudios e investigaciones en Río Turbio (Argentina). Estas dos ciudades, de países diferentes en nivel de desarrollo, poseen características geográficas y climáticas similares, pero opuestas en acceso a TIC y recursos sociales y de infraestructura. Mientras que en Montreal el citado grupo de personas se integra y moviliza en lugares públicos (restaurantes, galerías comerciales, universidades, bibliotecas, parques, entre otros) a través de vehículos especiales diseñados para su traslado (escúter), automóviles o medios públicos de transporte adaptados, ciudades subterráneas, entre otros, en Río Turbio, Argentina, los medios públicos de transporte no han sido preparados para tal fin, existiendo también cuestiones culturales que evidencian su falta de integración (ejemplo: rechazo de los taxis a trasladar discapacitados con sillas de ruedas, educación especial y no integración directa en la escuela pública, entre otros). El problema es complejo porque las desigualdades sociales y de ingresos generan falta de acceso a TIC donde más se necesitan.
} 


\subsection{Marco legal}

La citada Convención cristaliza un enfoque social de la discapacidad, estableciendo en su artículo 1:

“... promover, proteger y asegurar el goce pleno y en condiciones de igualdad de todos los derechos humanos y libertades fundamentales por todas las personas con discapacidad, y promover el respeto de su dignidad inherente. Las personas con discapacidad incluyen a aquellas que tengan deficiencias físicas, mentales, intelectuales o sensoriales a largo plazo que, al interactuar con diversas barreras, puedan impedir su participación plena y efectiva en la sociedad, en igualdad de condiciones con las demás"13.

Al respecto, una vía de reparación que promueve esta Convención es establecer la obligatoriedad, mencionada anteriormente, de: 1- ajustes razonables (remoción de barreras del entorno, incluyendo herramienta especial o la provisión de asistencia o apoyo a una persona con discapacidad en su lugar de trabajo $)^{14}$ y 2- la accesibilidad, que implica el tratamiento estructural y puede abarcar desde entornos físicos hasta el diseño y aplicación de una política pública determinada (diseño inclusivo). El diseño universal no excluirá las ayudas técnicas cuando se necesiten (Art. 2).

El enfoque social de la discapacidad - representado por la Convención - acierta en resaltar que la misma no puede escindirse del entorno social en que se desarrolla la vida de la persona (entramado social y dimensión afectiva familiar). Las barreras físicas, ambientales, procedimentales y comunicacionales, así como también los prejuicios y estigmas hacia la diversidad funcional de las personas, muestran que el problema no es individual. Esto implica brindar un criterio claro que atienda las necesidades y capacidades básicas para el desarrollo del ciudadano participativo.

\subsection{En la práctica}

Existe un abismo entre lo regulado en el plano jurídico y las estadísticas internacionales sobre la situación de vulnerabilidad de los "discapacitados" en sociedades no igualitarias, mostrando que las estipulaciones legales de derechos humanos se han convertido sólo en una expresión de intención (Morena, 2012). Según el Informe Mundial sobre la Discapacidad (2011) de la Organización Mundial de la Salud y el Banco Mundial, existen en el mundo alrededor de 1000 millones de personas con algún tipo de discapacidad. En países de bajos ingresos, tres cuartas partes de las mismas son mujeres, quienes ven agravada su situación de vulnerabilidad $^{15}$. Estos hechos evidencian la falta de políticas públicas que tomen en cuenta la interseccionalidad (género, etnia/raza, diversidad, clase social) citada anteriormente:

13. La Convención fue aprobada en el 2006 y ratificada por Argentina mediante ley 26.378 en el año 2008. Véase al respecto Convención por los derechos de personas con discapacidad: http://www.un.org/spanish/disabilities/default.asp?id=497.

14. La Convención, en su artículo 2, define por ajustes razonables “... las modificaciones y adaptaciones necesarias y adecuadas que no impongan una carga desproporcionada o indebida, cuando se requieran en un caso particular, para garantizar a las personas con discapacidad el goce o ejercicio, en igualdad de condiciones con las demás, de todos los derechos humanos y libertades fundamentales". Asimismo, define por diseño universal... "el diseño de productos, entornos, programas y servicios que puedan utilizar todas las personas, en la mayor medida posible, sin necesidad de adaptación ni diseño especializado" (Art. 2).

15. De acuerdo con Morena (2012), en Estados Unidos los hombres con discapacidad obtienen ingresos $55 \%$ más altos que las mujeres con discapacidad, mientras que sólo el $25 \%$ de las mujeres con discapacidad poseen empleo. 
"En el ámbito educativo, y de acuerdo con el Programa de Desarrollo de las Naciones Unidas (PNUD), la tasa de alfabetización de las personas con discapacidad es del $3 \%$, siendo sólo el 1 \% las mujeres y niñas alfabetizadas. El estudio efectuado por la Organización Human Rights Watch, señala que más de la mitad de las mujeres con discapacidad han sufrido algún tipo de abuso físico ${ }^{16}$. Las mujeres con discapacidad, a la vez son las más proclives a abusos en la atención médica, violatoria de los preceptos del consentimiento informado así como a esterilizaciones o abortos forzados, donde la paciente desconoce el tipo de intervención que se le efectuará así como el alcance de las mismas, descontando desde ya la falta de consentimiento prestado, en franca violación a todos los preceptos legales" (Morena, 2012).

En lo que respecta al acceso igualitario a TIC, en 2008 la Alianza Global para las TIC y el desarrollo de las Naciones Unidas (UNDESA GAID) lanzó la Iniciativa Global para TIC inclusivas (G3ict), proponiendo un kit de herramientas virtuales para el desarrollo de políticas públicas de accesibilidad digital para personas con discapacidad. De la misma manera, la Unión Internacional de Telecomunicaciones recomienda: implementar políticas para asegurar derechos y uso de G3ict como guía, reducir costos de acceso a banda ancha y servicios, implementar políticas para capacitar a personas con discapacidad, establecer fondos especiales para financiar iniciativas que promuevan las TIC para discapacitados y desarrollar estándares globales que garanticen la interoperabilidad de las infraestructuras e internet. A nivel de América Latina, la eLAC2010 "fomenta las TIC de calidad, asegurando el acceso sostenible de las mismas a personas con discapacidad y apuntando a su real inserción social, educativa, cultural y económica" (Meta 11) ${ }^{17}$.

A pesar de estas declaraciones, la disparidad de acceso a TIC y TA es muy grande en América Latina (entre países y en el interior de los países). Mientras que a nivel internacional se dispone de $\mathrm{TA}^{18}$ (lectores de pantalla, software de texto a voz y de voz a texto, software de reconocimiento óptico de caracteres y magnificadores, entre otros). Estos dispositivos son inasequibles para la mayoría de las personas con capacidades diversas en nuestros países.

De acuerdo con la UNESCO, esto ocurre porque la mayoría de los países latinoamericanos no cuenta con un marco regulatorio fuerte en torno a la implementación de las $\mathrm{TIC}^{19}$. El informe citado también señala que los mayores obstáculos para las personas con capacidades diversas y sus organizaciones son la falta de políticas públicas que articulen los ámbitos educativo, laboral y social; el marco legal débil para la defensa de los derechos; la confusión entre organizaciones de personas con discapacidad y organizaciones prestadoras de servicios; el desconocimiento de derechos y de mecanismos de exigibilidad, y las pocas posibilidades de acceso a TIC y TA. Todo ello contribuye a la invisibilidad de los ciudadanos con capacidades diversas.

16. Cursiva de la autora.

17. Se propone desarrollar proyectos, investigación y difusión de las TIC para la educación especial, desarrollar e impulsar la utilización de las TIC como herramientas para las personas con discapacidades, impulsar el uso de las herramientas TIC aplicadas a los ejes de desarrollo, formular planes para asegurar el acceso para personas con discapacidad y recopilar buenas prácticas de soluciones adecuadas por parte de instituciones o países, para difundir y replicar. Véase eLAC2010.

18. Las tecnologías de asistencia (TA) que mayor revolución han causado son el Jaws y la telefonía móvil.

19. La debilidad del marco, la falta de políticas o la incoherencia en el momento de armonizar las leyes dificultan el ejercicio de los derechos sociales. 


\subsection{Conclusión parcial del marco legal existente}

Según Díaz Velázquez (2010), la igualdad en la capacidad jurídica de las personas con discapacidad no es suficiente en tanto que no se actúe en la estructura social de tal manera que se atenúen y eliminen las barreras (materiales y simbólicas) que impone el entorno y las situaciones de discriminación que prevalecen. La ausencia de políticas públicas genera espacios llenos de barreras físicas y ambientales, procedimentales y comunicacionales, así como también prejuicios y estigmas hacia la diversidad funcional de las personas.

Si bien coincidimos con las afirmaciones anteriores, el punto que nos interesa resaltar aquí es que la estigmatización, que conlleva la desigualdad absoluta y relativa ${ }^{20}$ en el acceso a TIC y genera nuevas enfermedades psicosociales que deben preverse y atenderse en políticas de salud pública. Abocarse a investigar el problema de capacidades diversas en tanto cuestión de derechos humanos situados es entender que aquellas prácticas excluyentes vulneran de una manera especial tanto a grupos con capacidades diversas en el interior de los países en general como a grupos en países "no desarrollados" en el contexto de la comunidad internacional (brecha de acceso a TIC y TA para personas con capacidades diversas), afectando su salud psicosocial. El daño que el no acceso produce en su salud constituye un problema de justicia y una deuda social hacia las personas con capacidades diversas, el cual merece ser investigado y profundizado por la complejidad de factores que intervienen en la igualdad en salud. En este sentido, seguidamente analizaremos en qué medida las teorías filosóficas de justicia abordan el problema en su complejidad.

\section{Marcos filosóficos}

Las teorías de justicia inspiradas en el contrato social, como la teoría de justicia rawlsiana (Rawls, 1971), parten del supuesto de que sólo participarían del contrato los "miembros plenamente cooperantes de la sociedad, a lo largo de una vida completa". Si el acuerdo de cooperación es el beneficio mutuo, los participantes querrán juntarse con personas de cuya cooperación esperan obtener algo, no con aquellas que requieran atenciones inusuales y costosas. El presupuesto es que si esas personas no contribuyen al producto social y, por tanto, reducen el bienestar del conjunto de la sociedad, no pueden considerarse como miembros plenos en este nivel del contrato social (sería una cuestión de justicia especial a determinarse a posteriori del contrato social) ${ }^{21}$.

\footnotetext{
20. Por otra parte, el acceso al conocimiento y a la tecnología genética determina, en algunos casos, la posibilidad de prevención de la discapacidad por acceso anticipado a la información y la posibilidad de intervenir en el medio (epigenética). Véase De Ortúzar (2016).

21. Rawls no niega la inclusión de los ciudadanos con deficiencias atípicas, pero sostiene que debe resolverse en un estadio ulterior (justicia especial) por tres razones: 1- La primera deriva de su doctrina de los bienes primarios. Incluir a las personas con discapacidades pondría un obstáculo a la simple y directa vía para medir quiénes son los más desfavorecidos de una sociedad. 2- La segunda está relacionada con su adhesión a la tradición del contrato social. La relativa rareza de las deficiencias no "normales" implicaría introducir adaptaciones caras y complicadas en los lugares públicos de trabajo si queremos que sean plenamente accesibles para las personas con esa clase de deficiencias. En general, los gastos superarían por mucho los beneficios de la productividad económica resultante de una plena inclusión de esas personas. 3- La tercera y última razón afirma que la causa de salir del estado de naturaleza tiene que ver con los beneficios típicamente económicos que se obtienen de la cooperación mutua incapaz de ser lograda completamente por las personas con deficiencias atípicas. Véase Rawls (1971) cfr. Rawls (1995). Para Nussbaum, si se logra que las partes en la posición original no sepan si sufren o no alguna deficiencia, entonces, y sólo entonces, los principios resultantes serán verdaderamente equitativos. Véase sobre el tema Nussbaum (2007: 278).
} 
Al respecto, Nussbaum (2007), quien defiende una concepción de justicia política basada en derechos humanos $^{22}$, considera que dicho presupuesto es falso debido a que: 1- las personas con deficiencias y discapacidades no son improductivas; 2- las personas con discapacidades contribuyen de muchas maneras a la sociedad cuando ésta crea las condiciones que se lo permitan. Por ello, de los diferentes grados de discapacidad no se sigue la improductividad de la persona, como así tampoco la irreversibilidad o perpetuidad de la misma porque ésta depende, en gran medida, del entorno social. Y en este sentido, el acceso a TIC y TA es vital, especialmente en regiones pobres, para promover la citada igualdad.

Desde lo psicosocial, Nussbaum señala que la empatía y la vulnerabilidad son dos componentes centrales para poder reconocer al otro también como un ser frágil y necesitado, a la vez que capaz de cooperar en lo social, internalizando expectativas y normativas de sus compañeros para poder ser reconocidos como miembros de la cooperación social ${ }^{23}$. Por lo tanto, exige el acceso de aquello que hace posible una vida acorde con la dignidad humana, esto es: a los ciudadanos con capacidades diversas debería promoverse su salud, su educación y la plena participación en la vida social y, a ser posible, en la vida política. La citada autora propone organizar el trabajo de asistencia de los cuidadores de las personas dependientes (asistencia humana y financiera) y brindar oportunidades para el empleo satisfactorio a través de su capacitación y del uso de TIC y TA.

$\operatorname{Sen}^{24}(1995,2011)$ va un paso más allá de Nussbaum con su crítica a la rigidez de los bienes primarios rawlsianos. Sería muy difícil realizar comparaciones interpersonales porque las personas no son todas iguales y requieren distintas cantidades de un bien para lograr un bienestar similar. Los bienes son medios para lograr la libertad pero no la libertad misma. Por ello, propone centrarse en la variabilidad subjetiva y no en la igualdad de recursos (Sen, 2004: 2). Dicha propuesta se aplica muy bien a la discapacidad, entendida desde un enfoque social ${ }^{25}$.

El uso que podemos dar a un determinado nivel de rentas depende de algunas circunstancias. Sen identifica al menos cinco fuentes de diferencias entre nuestra renta real y las ventajas (el bienestar y la libertad) que obtenemos de ellas:

\footnotetext{
“1) Heterogeneidad personal: las personas tiene características físicas diferentes de acuerdo a la edad, el sexo, la propensión a enfermarse, etc. La compensación necesaria para contrarrestar las desventajas varía y, además, algunas desventajas no pueden ser compensadas con una transferencia de renta.
}

2) Diversidad relacionada con el medio ambiente: el clima, por ejemplo, puede influir en lo que obtiene una persona de un determinado nivel de renta.

\footnotetext{
22. A pesar de las críticas a Rawls, a nivel de justicia social Nussbaum (2007) extiende también la base del contrato social a nivel universal, es decir, no rompe con el contractualismo al dejar un espacio para el acuerdo razonable (consenso entrecruzado).

23. El requerimiento de entender y aplicar una concepción pública de la justicia demanda potenciar la capacidad de ponerse en el lugar del otro y de esa forma comprender tanto los potenciales beneficios como las necesarias cargas que deben asumirse. Para ello, el contar con un desarrollo de sentimientos empáticos puede ser un camino apropiado, al igual que el enfatizar el reconocimiento de la propia vulnerabilidad. La literatura, por ejemplo, es y ha sido un medio idóneo para ello, debido a que las narraciones tienen como rasgo distintivo el ampliar el mundo del lector y permitirle, a través de la imaginación, entrar en el de los protagonistas. Véase Nussbaum (2010: 199).

24. El citado autor critica a Rawls por no tener en cuenta las diferentes capacidades de cada uno para convertir los mismos recursos en beneficios. Pero Rawls tampoco tiene en cuenta situaciones de pobreza en su teoría ideal. Sen, al focalizar en el desarrollo de capacidades conectadas con las oportunidades reales que tienen las personas para el efectivo ejercicio de la libertad, justifica una propuesta apropiada como primera respuesta al problema de igualdad de los ciudadanos con deficiencias y discapacidades.

25. Para Sen (2004) el desarrollo puede concebirse como un proceso de expansión de las libertades reales de las que disfrutan los individuos.
} 
3) Diferencias de clima social: se trata de las condiciones sociales, incluidos los sistemas de enseñanza pública, el nivel de delincuencia, violencia, epidemiología y contaminación.

4) Diferencias entre las perspectivas relacionales: los bienes que exigen las costumbres de un lugar pueden variar de un lugar a otro. Por ejemplo, para poder "aparecer en público sin sonrojarse" puede ser necesario vestir mejor, consumir otros bienes visibles en las sociedades más ricas que en las más pobres.

5) Distribución dentro de las familias: de las reglas de distribución que se utilicen dentro de la familia pueden depender los logros y las dificultades económicas de sus miembros" ${ }^{26}$.

Estas causas de diferencias entre las rentas hacen que el nivel de renta no sea una guía muy eficiente del bienestar, señalando las limitaciones de la conversión, esto es:

"Si el fin es centrar la atención en las oportunidades reales del individuo para alcanzar sus objetivos habría que tener en cuenta no sólo los bienes primarios que poseen las personas sino también las características personales relevantes que determinan la conversión de los bienes primarios en la capacidad de la persona para alcanzar sus fines" (Sen, 2000: 99).

En el caso que nos ocupa, las personas con capacidades diversas necesitan más recursos (ayudas técnicas o apoyos personales) para garantizar un bienestar aceptable. Por ejemplo, mayores gastos para la vida diaria en sueldos de apoyos personales o adaptaciones técnicas de su hogar, entre otros.

Como hemos señalado anteriormente, los factores de conversión son individuales, sociales y/o medio ambientales. Por ejemplo, para alcanzar la función de ser capaz de moverse libremente, el recurso requerido dependerá en parte en la habilidad de mover las piernas, o del tipo de terreno, o de la presencia de medios de transporte. Lo importante - según Sen- no es si la persona se moviliza en un auto o en una mula o en una bicicleta, sino si es capaz de llegar a donde quiera llegar (volveré sobre este punto más adelante). El citado autor reconoce la importancia de ejercer la libertad para escoger entre diferentes estilos y modos de vida (2010: 257).

Pero una persona con alguna de sus capacidades funcionales reducida o limitada, se encuentra con limitaciones en la ganancia, es decir, limitaciones para poder alcanzar los recursos y bienes primarios. Por ejemplo, dificultades para conseguir empleo e ingresos menores por su trabajo, como ocurre con el caso de las mujeres - mencionado anteriormente- que sufren triple discriminación: por su condición de mujeres pobres con capacidades diversas. Por lo tanto, es necesario actuar brindando acceso igualitario a TIC y TA de acuerdo a necesidades, como así también apoyo especial y adaptación del ambiente en pos de que las personas con capacidades diversas puedan integrase socialmente y desarrollar sus propias capacidades. Asimismo es importante actuar tanto en el nivel simbólico como estructural, para evitar cualquier tipo de discriminación en oportunidades de educación, empleo e inclusión social general. En algunos casos, las políticas de discriminación positiva son las indicadas para promover dicho acceso igualitario, pero no suficientes.

26. Véase Sen (2000: 70-71) 


\subsection{Conclusión parcial de los marcos filosóficos}

La concepción de la igualdad de capacidades citada tiene la ventaja de ampliar la visión qué implica la justicia social: no se trata sólo de distribuir bienes materiales, sino también de considerar la variabilidad subjetiva (capacidades) para esta distribución. Por otra parte, si queremos alcanzar el ejercicio de la libertad de todos, es importante paliar la desigualdad social inicial ${ }^{27}$. La pobreza es redefinida justamente como la privación de las capacidades, y no desde la clásica tradición económica, la cual mide la pobreza por el consumo.

Esta visión tiene la fortaleza de no descuidar los elementos simbólicos relevantes para eliminar las barreras hacia las capacidades diversas, como lo es promover la empatía. Como bien señala Díaz Velázquez (2010,115-135):

"Sen (y Nussbaum) ven obligatorio e ineludible que todo sistema social que se inspire en los principios de justicia equitativa y distributiva, desarrolle las herramientas y los recursos básicos para que las personas con discapacidad puedan alcanzar un bienestar aceptable. No obstante,... no siempre es una cuestión de aplicación de apoyos personales y ayudas técnicas, sino que muchas veces éstas no son suficientes y se necesita incidir en las barreras (estructurales o simbólicas) del entorno social”.

En lo que respecta a Sen, su aporte especial se encuentra en considerar la diversidad de relaciones con el medio ambiente (por ejemplo, condiciones climáticas adversas que generan aislamiento), como con las condiciones sociales (falta de educación, vivienda apropiada, entre otros). Sin embargo, estas últimas -las condiciones sociales - parecen ser evaluadas de un modo fijo, estático, y no dinámico. Su última referencia a la perspectiva relacional apunta a la variación comparativa de las costumbres en el mundo y a la necesidad de compensarlas para que no exista humillación por falta de igualdad (ejemplo: necesidad de vestimenta elegante como norma social para poder hablar en público en países diferentes al nuestro). Sin embargo, las diferencias sociales más importantes, producto de las desigualdades sociales, no se limitan sólo a éste aspecto de la cultura, sino que conllevan el debilitamiento de las relaciones sociales como consecuencia de las diferencias económicas, de estatus, planteando relaciones de humillaciones y sometimiento que afectan la calidad de vida y salud de una gran parte de la población, y muy especialmente de los más débiles. Las relaciones pobres inciden negativamente en las oportunidades de los más vulnerables (entendiendo a la vulnerabilidad como categoría relacional desde un enfoque interseccional - género, raza/etnia, clase social, edad, diversidad- que tiende a visibilizar las asimetrías de poder).

El problema de equidad en capacidades diversas no resulta exclusivamente de un atributo individual o una tragedia personal, sino de una relación social, que posee grados y no implica la desigualdad permanente. Por ejemplo, en el caso de la movilidad - de acuerdo con Sen - lo importante no es el medio sino dónde quiere llegar la persona. Pero si una persona en silla de ruedas de una zona pobre y aislada tiene que trasladarse en una mula con un carro para acudir a la escuela o a su trabajo, mientras que otras personas en su misma situación acceden al autobús adaptado o a autos especiales para efectuar un cómodo traslado, entonces esa persona, aun cuando llegue a su meta, sufrirá la desigualdad relativa, es decir una estigmatización social por diferencia de estatus que afectará su salud, al ser consciente de encontrarse en una situación de inferioridad en relación con los otros. Su libertad de escoger se verá afectada y sufrirá por estatus bajo. 
Nussbaum establece un umbral mínimo de las capacidades. Se trata de alcanzar esos mínimos por debajo de los cuáles no existe una vida digna. Pero si una persona con capacidades diversas accede a una vivienda digna, y su vecino construye una mansión que brinda diferentes oportunidades tecnológicas de movilización, visión, audición, entre otras; la persona con capacidades diferentes sufrirá la desigualdad relativa vivenciando su estatus diferencial (a pesar de satisfacer el umbral mínimo). Atender a las desigualdades absolutas a través de la igualdad de capacidades permite paliar las grandes diferencias sociales de estatus, pero no elimina desigualdades relativas. He aquí mi crítica y mi reconocimiento parcial a la teoría de SenNussbaum, señalando la importancia de evaluar también el impacto de las desigualdades relativas en salud.

\section{El aporte de la desigualdad relativa en relación al acceso igualitario a TIC en personas con capacidades diversas}

\subsection{Desigualdad absoluta y salud}

Como hemos señalado anteriormente, las teorías de justicia - aun las aplicadas a salud - suelen centrarse en la desigualdad absoluta. La desigualdad absoluta afecta al individuo más allá de si él mismo es consciente o no de la desigualdad (Wilkinson, 2005). Por ejemplo, la pobreza absoluta equivale a carecer de la satisfacción de necesidades básicas (comida, abrigo, entre otras). Asimismo, puede considerarse que el daño ambiental - por ejemplo agua contaminada, falta de cloacas o polución, entre otras - constituye un factor externo y determinante social material básico que afecta a la salud. Estos determinantes deben ser prevenidos, controlados o evitados porque generan daños directos. La citada desigualdad puede modificarse con la simple intervención material (satisfacción de necesidades y capacidades básicas y/o protección medio ambiental). En este sentido, uno debería poder exigir al Estado que cumpla con los derechos humanos establecidos a nivel internacional y nacional en nuestra Constitución (Argentina) donde se establece el derecho a la salud (aun cuando éste no se reduzca a las necesidades básicas).

Ahora bien, si existen los recursos para terminar con la pobreza absoluta, y si existen derechos que protegen contra el daño ambiental y que defienden positivamente la salud, entonces el problema no es material ni legal, sino político y distributivo. Los que sufren problemas de salud y daños ambientales se encuentran en una condición de vulnerabilidad, es decir, su situación supone una asimetría de poder y diferencias que hacen que sólo algunas personas gocen de libertad real. Pero muy pocas teorías de justicia sostienen quiénes son los responsables de evitar dicha situación de asimetría que genera la desigualdad y la pobreza: "está claro que muchas personas están siendo usadas como medios, aunque no está claro quién tiene el deber de evitarlo..." (Nussbaum, 2007: 276-277) ${ }^{28}$, siendo necesario determinar principios de justicia global y responsables (volveré más adelante sobre este punto).

En el tema que nos ocupa, el acceso igualitario a TIC y TA, la desigualdad absoluta se genera por la falta de acceso a TIC y la no intervención en el entorno para personas con capacidades diversas en zonas aisladas, inhóspitas y pobres. En términos generales, el acceso a TIC es una necesidad esencial para toda la comunidad de zonas vulnerables, pero más aún para el grupo de personas con capacidades diversas, 
debido a que a su desigualdad inicial y social se suma la desigualdad climática y geográfica que incide especialmente en sus posibilidades de manejarse en el entorno. Para los discapacitados de dichas comunidades el acceso a TIC es una prioridad porque constituye el único medio de acceso a la atención de la salud especializada (telemedicina) y a la educación continua (teleeducación), como también constituye el único medio para relacionarse a distancia cuando existen bajas temperaturas y se produce el aislamiento por condiciones climáticas y geográficas (De Ortúzar, 2012). Su aplicación supone capacitación, apoyo especial, herramientas específicas - TA - requeridas para el acceso a empleos y medios de transportes adecuados a las condiciones geográficas adversas, eliminación de barreras simbólicas y, por sobre todas las cosas, eliminación de las diferencias de estatus en las comunidades del sur (teniendo en cuenta género, etnia, clase social, diversidad).

El acceso a TIC y TA en dichos casos no es sólo responsabilidad de políticas públicas regionales que incidan en el entorno social, aunque las mismas deben ser prioridad del Estado. También inciden en la desigualdad de acceso los acuerdos internacionales que, lejos de compensar desigualdades entre países generadas por injustas razones históricas, imponen sus políticas globales a nivel local a través de asimétricas negociaciones como el mencionado ADPIC - segundo determinante social global de la salud- (Filho, 2001; Pogge, 2005). Este punto ha sido reconocido parcialmente por las teorías de justicia de Nussbaum y Pogge, señalando la responsabilidad del orden internacional actual, de los organismos internacionales, de los países ricos y de los ciudadanos de países desarrollados, entre otros.

\subsection{La desigualdad absoluta genera desigualdad relativa en salud}

Ahora bien, los estudios recientes ${ }^{29}$ sobre los determinantes sociales de la salud han evidenciado que el tipo de desigualdad que afecta principalmente nuestra calidad de vida no es simplemente la desigualdad absoluta (necesidades básicas/ingresos absolutos), sino la desigualdad relativa (diferencia de estatus/ingresos relativos). Es decir, podemos tener una mejor calidad de vida con un ingreso menor (ingreso relativo), lo relevante es si la sociedad es igualitaria. Según Wilkinson y Pickett, para el bienestar no es necesario tener más ingresos, sino vivir en el marco de una sociedad donde no existan grandes disparidades, donde la riqueza esté distribuida equitativamente. La desigualdad relativa resulta de la desigualdad social y ocasiona diferencias de estatus. Las personas que se identifican con un nivel de estatus social bajo (ingreso relativo) tendrán una vida de menor calidad que los de nivel superior, padeciendo una mayor propensión a enfermedades psicosociales por estrés crónico, sufriendo violencia, nuevas fobias, enfermedades de países desarrollados - cáncer, enfermedades cardíacas, drogadicción, obesidad-, entre otros factores que harán su vida más corta (Wilkinson, 2005) ${ }^{30}$.

La desigualdad relativa también afecta a los grupos discapacitados en sociedades no igualitarias y, comparativamente, a los grupos pobres de la comunidad internacional de "discapacitados". En el caso de acceso a TIC y TA, resulta claro que, aun cuando las personas con capacidades diversas logren satisfacer sus

29. Me refiero a los estudios de la epidemiología, psicología, antropología o neurociencia, entre otras. Al respecto, ver respectivamente los siguientes trabajos: 1. Easterlin, 1974, 2005; 2. Haidt, 2006; 3. Erik Olin Wright, 1997; 4. Wilkinson, 2005 y 2009 , entre otros.

30. Los lazos sociales (amigos, familia, pertenencia a la comunidad) son precisamente los que protegen al hombre contra el estrés crónico de la sociedad tecnológica, aunque no evitan las desigualdades. Por el contrario, el individualismo del sistema promueve la competitividad, creando una sociedad disfuncional y anticooperativa por ruptura de relaciones de confianza, seguridad y relaciones de amistad. El mercado se constituye en el único elemento "homogeneizador" de los ciudadanos: el consumismo ("ciudadano consumidor"). Sobre éste punto véase De Ortúzar (2016). 
necesidades básicas, también son conscientes de la diferencia de estatus que se da por el acceso a TIC y TA. Esta diferencia nace de la desigualdad social y de las dificultades que existen en la conversión y en la ganancia, lo cual exige un estudio interseccional (etnia, género, diversidad, clase social), como así también consideraciones no estáticas, sino dinámicas, de los determinantes sociales (no fijos ni individuales), las relaciones climáticas (evaluación de la relación con el medio ambiente) y las relaciones asimetrías de poder en lo que respecta a la cultura (libertad o colonización). Por ejemplo, si consideramos el caso de acceso a TIC en nuestra región de personas con capacidades diversas, sabemos que el mismo está condicionado por cuestiones de mercado. Es decir, la innovación en TIC y el acceso a las mismas sólo puede ser posible en nuestra región en las ciudades más ricas (Thery, H. y De Mello-Thery, 2012) porque la investigación pública no tiene financiamiento para este tipo de necesidades (Brecha 10/90, OMS ${ }^{31}$ ), existiendo sólo un mercado muy selectivo no regional - amparado también por el ADPIC - . La relación entre desigualdad social y desigualdad de acceso a TIC y TA es un ejemplo de desigualdad relativa padecida por grupos con capacidades diversas en regiones pobres. Dicha desigualdad crea estigmatización y trato diferencial en un mismo país y entre países, generando no sólo la misma discapacidad por entorno social (enfoque social), sino también por la conciencia de diferenciación en el estatus de ciudadanos por regiones ${ }^{32}$. En otras palabras, acceden a TIC y TA los "usuarios y/o consumidores" que puedan pagar el precio del producto, y no los ciudadanos que los necesiten por cuestiones de salud, no existiendo políticas públicas - regionales e internacionales - para su justa distribución. Por ello, las diferencias sociales se traducen en nuevas enfermedades psicosociales también para éste grupo, que se suman a la falta de acceso igualitario a TIC y TA para capacidades diversas.

Si consideramos la discapacidad desde el enfoque social, entonces es importante reconsiderar nuestras concepciones de justicia, para que éstas se centren en las relaciones entre los ciudadanos democráticos y empáticos ${ }^{33}$, y no sólo en la distribución de bienes o en el estudio de condicionantes sociales como algo fijo y dado. Tampoco se trata de continuar con la paradójica convivencia de un modelo hegemónico que habilita al mercado a ofrecer variedad de productos por perfil individual, sin evaluar si éste es adaptable al entorno social y beneficia la integración social, y/o profundiza las diferencias. Se trata de investigar las complejas relaciones que se dan entre el individuo, la familia, el grupo social, la sociedad y las condiciones de trabajo, buscando transformar estas condiciones que no generan cooperación y fortaleciendo las relaciones sociales que permiten la integración. En este sentido, coincido con Sen y Nussbaum en algunos puntos relativos a diferencias materiales y simbólicas, pero me diferencio de dichos autores al sostener que la desigualdad más importante en el acceso a TIC y TA de los discapacitados no es sólo la absoluta, sino también la desigualdad relativa derivada de la misma.

31. La brecha 10/90 reconocida por la OMS en investigación denuncia la creciente desigualdad - a nivel de acceso a medicamentos e investigación- entre países industrializados y países en vía de desarrollo: el 90 \% de los esfuerzos y recursos destinados a la investigación biomédica se destinan a problemas que afectan tan sólo al $10 \%$ de las prioridades en salud pública a nivel global y, de igual forma, solo se destina el $10 \%$ de dichos recursos de investigación a solucionar problemas de salud que afectan al $90 \%$ de la población mundial. A partir del conocimiento público de esta desigualdad, se crea en 1998 el Foro Mundial sobre Investigaciones Sanitarias. El propósito fundamental del Foro Mundial se orienta a fomentar que las iniciativas de investigación se centren en las enfermedades que representan la mayor carga mundial de morbilidad, a mejorar la asignación de los fondos de investigación y a facilitar la cooperación entre asociados de ambos sectores, público y privado. Es innegable que las inversiones mundiales en investigación no abordan satisfactoriamente las cuestiones de salud que más afectan a los países pobres. Una mayor financiación es condición necesaria, pero no suficiente, para mejorar los resultados de salud. Existe, además, la urgente necesidad de elaborar programas nacionales de investigación que aborden las prioridades locales en materia de salud para desarrollar a largo plazo la capacidad investigadora nacional e internacional, y de idear mecanismos que mejoren la coordinación entre las prioridades de investigación y su sostén económico. Véase al respecto: Organización Mundial de la Salud (2001).

32. En las regiones ricas las innovaciones en TIC y TA están presentes tanto en el mercado como en políticas de investigación y políticas públicas. Sin embargo, en las regiones pobres no se investiga ni se accede a las mismas.

33. Excepto en la atención y cobertura de las necesidades esenciales y específicas, que hace a lo singular. 
5.3. Conclusión parcial del aporte de la desigualdad relativa en relación con el acceso igualitario a TIC en personas con capacidades diversas

Repasando los pasos positivos dados en el análisis de la desigualdad, considero que:

1. Resulta crucial, en pos de la igualdad, comenzar actuando sobre las condiciones de pobreza, entendida como privación de capacidades y falta de riqueza social, y no considerando la misma exclusivamente desde el punto de vista monetario o de consumo.

2. Para el desarrollo de capacidades no debemos tener en cuenta sólo la distribución de recursos sino también la variabilidad intersujetiva - adecuación de recursos al sujeto/conversión-. Pero, como es claro en el caso de la discapacidad, se debe actuar sobre el entorno social dinámico que afecta al mismo, eliminando la desigualdad (no sólo a nivel individual). Por ejemplo, se debe actuar sobre el espacio social, transformar la movilidad (modificación del transporte), brindar asistencia especial/acceso a TA, garantizar que los ingresos de personas con discapacidades y sus oportunidades de empleo sean equitativas o fomentar el acceso a la educación continua, entre otros.

Estos dos primeros puntos contribuirían a compensar las barreras estructurales que hacen a la discapacidad. Por otra parte, coincido con Nussbaum en que no deben descuidarse las barreras simbólicas, esto es:

3. Trabajar la cohesión social y la cooperación, que incide en lo simbólico, y muy especialmente la necesaria construcción de lazos que permitan desarrollar la empatía hacia los más vulnerables en un contexto de reconocimiento de la ciudadanía, de reconocimiento de la democracia y de reconocimiento de los derechos humanos. El concepto de ciudadanía se contrapone aquí al concepto de "usuario", criticando la clasificación de "perfiles de usuarios con capacidades diversas", ya que estos perfiles marginan del ejercicio del derecho a la salud a los que no pueden pagar.

Estos temas reseñados han sido mencionados transversalmente en las teorías de justicia, debiendo profundizarse en gran medida para la comprensión de la diversidad de capacidades por parte de la comunidad (empatía y vulnerabilidad). Es central eliminar de raíz las barreras simbólicas, los prejuicios en torno a la diversidad de capacidades y considerar la interseccionalidad (género, raza, etnia, clase social) en el abordaje de las mismas para construir la figura del ciudadano democrático y comprometido, y no de un simple "usuario de mercado".

Por último, no podemos descuidar, en función de su importancia creciente, la desigualdad relativa; y cómo las consiguientes diferencias sociales de acceso generan humillación y estigmatización de los grupos de personas con capacidades diversas de regiones pobres. Esta vulnerabilidad es producto de la desigualdad social por falta de políticas distributivas a nivel regional e internacional, por las consiguientes diferencias de estatus por asimetrías de poder y por la ausencia de desarrollo de empatía con el vulnerable ante la imposición de una concepción de mercado (el usuario que paga accede). Parecería que ya no están en vigencia los derechos del ciudadano, los derechos humanos. Pero, ¿quiénes son los responsables de esta situación? Los tratados internacionales (ADPIC), los laboratorios y sus privilegios en la financiación de investigación en prioridades (Brecha 10/90, OMS), la complicidad de gobiernos locales y la falta de políticas públicas; todos inciden en la desigualdad de acceso a TIC y TA regional e internacional, afectando estas desigualdades sociales a los grupos con capacidades diversas. Este último punto, nos lleva a sostener la necesidad de: 
4. Actuar sobre las diferencias de acceso a TIC y TA (determinante social global) generadas por ADPIC y por la distribución inequitativa en las investigaciones sanitarias regionales (Brecha 10/90, OMS), y proponer la evaluación de políticas públicas integrales que tengan en cuenta los determinantes sociales para el uso social de $\mathrm{TIC}^{34}$, promoviendo un abordaje interseccional (género, etnia, clase social, diversidad, interculturalidad), integral y situado (uso social adaptado y cultural) en pos de paliar la desigualdad relativa que origina nuevas enfermedades psicosociales en grupos vulnerables de personas con capacidades diversas.

\section{Conclusión}

A modo de cierre, la defensa de los derechos humanos de las personas con capacidades diversas y la defensa específica de su acceso igualitario a TIC y TA para hacer su vida - en algunos casos - posible, exige un marco legal armónico y fuerte, sustentado en una concepción de justicia social que atienda el problema actual de la desigualdad relativa, y políticas públicas acordes para su concreción. De acuerdo con Filho, y en relación con los determinantes sociales que constituyen el acceso a TIC, "estos bienes públicos tienden cada vez más a transformarse en bienes públicos globales, lo que obliga a que las políticas que estimulan su creación y distribución sean objeto de nuevos acuerdos institucionales de la comunidad internacional, incluyendo un nuevo papel de instituciones multilaterales tradicionales" (Filho, 2001: 409-412). Más allá del problema de fomentar nuevos acuerdos internacionales que protejan -y no comercialicen - los bienes públicos, conjuntamente con políticas regionales, me parece que no puede descuidarse el problema de fondo: las desigualdades sociales (internacionales y regionales) y los intereses del mercado generan una gran disparidad en la innovación y acceso a TIC y TA de las personas que más lo necesitan en regiones pobres (Brecha 10/90). Este problema de acceso a dichos bienes públicos (conocimiento/TIC/salud) no es sólo un problema de desigualdad absoluta. Es también un problema de desigualdad relativa con fuertes incidencias en la salud psicosocial de las personas con capacidades diversas.

En pos de enfrentar el mismo, es importante continuar trabajando en:

1. Crear nuevos marcos legales internacionales y regionales fuertes, armonizando los mismos para su efectivo cumplimiento, de modo que puedan traducirse en políticas públicas integrales que contribuyan a paliar la desigualdad relativa ${ }^{35}$ de nuestra sociedad tecnológica.

2. Promover el financiamiento público igualitario en innovaciones en TIC y TA de acuerdo a necesidades de personas con capacidades diversas, compensando - internacional y regionalmente - las disparidades en innovación y acceso generadas por financiamientos regionales diferenciales ${ }^{36}$.

34. Por ejemplo, a pesar del reconocimiento de derechos - Tratado de Marrakech - , existen restricciones reales de acceso a libros adaptados debido a leyes de propiedad intelectual, gracias a la falta de armonización de marcos, asimetrías de poder y diferencias de ingresos, lo cual ocasiona serias diferencias sociales que repercuten en la salud y posibilidades de vida de las personas con deficiencias visuales.

35. Por ejemplo, la inclusión de la discapacidad como tema transversal en la educación superior, la formación de docentes para atender la diversidad con conocimientos de TIC y TA, el diseño universal en áreas que competen al diseño de páginas web y sistemas operativos, programación y fabricación, entre otros. A nivel de barreras simbólicas, se trabaja con campañas masivas de información y sensibilización, planes educativos vertebrados por principios inclusivos y programas de inserción laboral e intercambios internacionales, alfabetización digital y capacitación docente con desarrollo de competencias profesionales vinculadas a TIC y TA.

36. El financiamiento permitirá mejorar la infraestructura, el acceso a tecnologías emergentes de acuerdo a necesidades y disminuir costos. Por 
3. Fortalecer los lazos sociales internacionales y regionales en la comunidad de personas con capacidades diversas, trabajando en la igualdad social para actuar colectivamente contra las diferencias de estatus de personas con capacidades diversas causadas por diferencia de acceso a TIC y TA regionales.

4. Educar en la empatía hacia el más vulnerable, reforzando los lazos simbólicos, los derechos humanos para la defensa de los derechos de ciudadanía vs. los perfiles de usuarios individuales y exclusivos intereses de los mercados.

5. Evaluar los efectos de estas desigualdades relativas en la salud psicosocial de las personas con capacidades diversas debido a que no sólo debe tenerse en cuenta su deficiencia, sino conjuntamente ésta con las barreras estructurales y simbólicas para su integración social. Es necesario también estudiar cómo las diferencias sociales en el acceso a TIC y TA generan nuevas enfermedades psicosociales. Estas no pueden ser ignoradas, demandando la evaluación temprana de las capacidades diversas con enfoque integral e interseccional (género, raza/etnia, clase social, diversidad) y promoviendo marcos legales armónicos, coherentes y fuertes, conjuntamente con políticas públicas integrales.

Una vez más, si queremos superar las barreras simbólicas y estructurales que sufren las personas con capacidades diversas, no podemos descuidar la desigualdad relativa de acceso a TIC y TA ocasionada por las desigualdades sociales, por desigualdades en financiamiento de investigaciones, por asimetría de poder y por las consecuentes diferencias entre grupos y entre países (ADPIC, Brecha 10/90). La conciencia de la existencia de dicha desigualdad genera estatus diferencial entre ciudadanos con capacidades diversas por regiones y grupos, dañando su salud, generando nuevas enfermedades psicosociales y atentando contra los derechos humanos reconocidos a nivel formal, pero sin correlato de armonización legal práctica para su ejercicio. Nos encontramos ante un panorama social -internacional y regional- que, aun en un tema tan delicado como lo es el acceso igualitario a TIC y TA de personas con capacidades diversas, parece negar los "derechos del ciudadano" y privilegiar en forma absoluta la libertad del "usuario".

ejemplo, en términos de costo y distribución, podría ser de mayor beneficio la consecución de: lectores de pantalla, digitalizadores de textos, comunicadores con sistemas de símbolos, Netbooks, Wifi gratuito en instituciones, software de texto a voz y de voz a texto. 


\section{Referencias bibliográficas}

Alaniz, M. et al. (2010): “Los Aportes de la Tecnología Informática a la Educación Especial. El caso de la Escuela No 9 Keoken de Río Turbio". Revista de Informes Científicos - Técnicos UNPA, 2 (3): 214-237.

Argentina. Ley 27.044, de 11 de diciembre, por la que se otorga jerarquía constitucional a la Convención sobre los Derechos de las Personas con Discapacidad, Boletín Oficial de la República Argentina, 22 de diciembre de 2014, núm. 33.035, pp. 3.

Argentina. Ley 26.378, de 6 de junio, por la que se aprueba la Convención sobre los Derechos de las Personas con Discapacidad y su protocolo facultativo, aprobados mediante resolución de la Asamblea General de las Naciones Unidas del 13 de diciembre de 2006, Boletín Oficial de la República Argentina, 9 de junio de 2008, núm. 31.422, pp. 1.

Braithwaite, J. (2009): "Disability and poverty: A survey of World Bank Poverty Assessments and implications". ALTER, European Journal of Disability Research, 3 (3): 219-232.

Breilh, J. (2013): "La determinación social de la salud como herramienta de transformación hacia una nueva salud pública (salud colectiva)". Revista Facultad Nacional de Salud Pública, 31: 13-27.

Canimas, J. (2015): “¿Discapacidad o diversidad funcional?”. Siglo Cero: Revista Española sobre Discapacidad Intelectual, 46 (2): 79-97.Final del formulario

De Ortúzar, M. G. (2016): "Responsabilidad social vs. Responsabilidad individual en salud”. Revista de Bioética y Derecho, 36: 23-36.

De Ortúzar, M. G. (2014): “'Gobernanza’ y ‘Gobernanza en salud’: ¿Una nueva forma de privatizar el poder político?”. OXÍMORA Revista Internacional de Ética y Política, 5: 63-86.

De Ortúzar, M. G. (2012): "Hacia la telemedicina en el MERCOSUR. Análisis ético comparativo de las normativas de Brasil y Argentina". RevistaeSalud.com, 8 (30): 1-17.

De Ortúzar, M. G. (2003): “Towards a universal definition of benefit sharing”, en Knoppers, B. M. (ed.): Population and Genetics: Legal and Socio-Ethical Perspectives. Leiden/Boston: Martinus Nijhoff Publishers.

Díaz Velázquez, E. (2010): "Ciudadanía, identidad y exclusión social de personas con discapacidad”. Política y Sociedad, 47 (1): 115-135.

Easterlin, R. A (2005): "Building a Better Theory of Well-Being”, en Bruni, L. y Porta, P. L. (eds.): Economics and Happiness: Framing the analysis. Oxford: Oxford University Press.

Easterlin, R. A (1974): "Does Economic Growth Improve the Human Lot?", en David, P. A. y Reder, M. W. (eds.): Nations and Households in Economic Growth: Essays in Honor of Moses Abramovitz. Nueva York: Academic Press.

Filho, A. P. (2001): "Inequities in access to information and inequities in health", Revista Panamericana de Salud Pública, 11 (5-6): 409-412.

Green, R. M. (1997): "Parental Autonomy and the Obligation Not to Harm One's Child Genetically". The Journal of Law, Medicine \& Ethics, 25 (1): 5-15.

Haidt, J. (2006): The Happiness Hypothesis: Finding Modern Truth in Ancient Wisdom. Nueva York: Basic Books. 
Morena, N. (2012): Mujeres con discapacidad en sociedades pobres: la triple discriminación (Trabajo final de la Especialización en Ciencias Políticas). Universidad de Quilmes, Argentina.

Nussbaum, M. (2010): Sin fines de lucro. Buenos Aires/Madrid: Katz Editores.

Nussbaum, M. (2007): Las fronteras de la justicia. Barcelona: Paidós.

Organización de las Naciones Unidas, ONU (2008): El derecho a la salud. Folleto informativo No 31. Ginebra: Organización Mundial de la Salud.

Organización de las Naciones Unidas, ONU (2006): Convención Internacional sobre los Derechos de las Personas con Discapacidad. Nueva York: ONU.

Organización Mundial de la Salud (2001): Macroeconomics and Health: Investing in Health for Economic Development (en línea). <http://www.who.int/macrohealth/action/sintesis15novingles.pdf>, acceso 17 de mayo de 2018.

Organización Mundial de la Salud y Banco Mundial (2011): Informe mundial sobre la discapacidad (en línea). <http://www.who.int/disabilities/world_report/2011/es/index.html>, acceso 17 de mayo de 2018.

Organización Mundial del Comercio (2003): Acuerdo de los Derechos de Propiedad Intelectual relacionados con el comercio (en línea). <https://www.wto.org/spanish/tratop_s/trips_s/trips_s.htm>, acceso 17 de mayo de 2018.

Palacios, A. (2008): El modelo social de discapacidad. Orígenes, caracterización y plasmación en la Convención Internacional sobre los Derechos de las Personas con Discapacidad. Madrid: CINCA.

Pogge, T. (2005): World Poverty and Human Rights. Cambridge: Cambridge University Press.

Rawls, J. (1995): Liberalismo político. México, D.F.: Fondo de Cultura Económica.

Rawls, J. (1995): Teoría de la Justicia. México, D.F.: Fondo de Cultura Económica.

Sen, A. (2011): La idea de la Justicia. Buenos Aires: Taurus.

Sen, A. (2004): "Why health equity?", en Anand, S. et al. (eds.): Public health, ethics and equity. New York: Oxford University Press.

Sen, A. (2000): Desarrollo y libertad. Barcelona: Planeta.

Sen, A. (1995): Nuevo examen de la desigualdad. Madrid: Alianza.

Sherlaw, W. et al. (2013): "Disabled people, inclusion and policy: better outcomes through a public health approach?". Disability and Society, 29 (3): 444-459.

Thery, H. y De Mello-Thery (2012): “Disparidades e dinâmicas territoriais no Brasil”. Revista do Departamento de Geografia, USP, Volume Especial 30 Anos: 68-91.

Vallejos, I. (2012): "Entre focalización y universalidad de las políticas con discapacidad: el enfoque de derechos", en Indiana Almeida, M. E. y Angelino, M. A. (comps.): Debates y perspectivas en torno a la discapacidad en América Latina. Paraná, Argentina: Universidad Nacional de Entre Ríos.

Wilkinson, R. (2005): The impact of inequality. Nueva York: The New Press.

Wilkinson, R. y Pickett, K. (2009): Desigualdad: un análisis de la (in)felicidad colectiva. Madrid: Turner Publications.

Wright, E. O. (1997): Class Counts: Comparative Studies in Class Analysis. Cambridge: Cambridge University Press. 